\title{
Association of Obstructive Sleep Apnea Indicators with Heart Failure
}

\author{
Faheem Handoo ${ }^{1}$, Yuyao Liu², Sonja G. Schütz ${ }^{3}$, Ronald D. Chervin ${ }^{3}$, Ivo D. Dinov² \\ ${ }^{1}$ Indiana University School of Medicine; ${ }^{2}$ Statistics Online Computational Resource (SOCR), \\ Department of Health Behavior and Biological Sciences, University of Michigan; ${ }^{3}$ Department \\ of Neurology, University of Michigan Medical School
}

Background: Obstructive sleep apnea (OSA) occurs when the airway is repeatedly blocked during sleep, resulting in frequent brief awakenings throughout the night. OSA has been found to increase the risk of many cardiovascular diseases, especially heart failure (HF). HF with reduced, preserved, and borderline ejection fraction (HFrEF, HFpEF, and $\mathrm{HFbEF}$ ) are three subtypes common in OSA patients. The aim of this study is to further explore the relationship between OSA and HF and the influence of specific OSA measures.

Methods: Electronic medical data was collected from health histories, echocardiograms, and polysomnography studies. Observations were sorted into three categories based on left ventricular ejection fraction: $\operatorname{HFpEF}(n=334), \operatorname{HFrEF}(n=77)$, and $\operatorname{HFbEF}(n=37)$. Multinomial logistic regression was then conducted to determine the relative risk of HFpEF and HFrEF from each variable as compared to the baseline $\mathrm{HFbEF}$.

Results: Pacemaker presence, previous stroke, BMI, and a measure of left ventricular dysfunction (LVD), called relative wall thickness, all raised the risk of HFpEF compared to $\mathrm{HFbEF}$, while another LVD measure, left ventricular end-systolic dimension, reduced it. These factors also increased risk for HFrEF, except for previous stroke and pacemaker presence, which were not significant. Relevant OSA metrices included average blood oxygen saturation and three measures of sleep apnea severity, named central apnea index, hypopnea index per hour, and the Epworth Sleepiness Scale (ESS). These all decreased relative HFpEF risk, other than ESS, which raised it.

Conclusions: As was expected, several standard HF predictors increased the risk of both types of HF. Surprisingly, few OSA indices had the same effect. This suggests that targeting specific OSA markers may not be effective in treating patients with any of these HF types. Future work could involve the influence of OSA and its indices on mortality, or the responses of these indicators to treatment, both topics with limited previous findings. 